\title{
Research Paper \\ The Relationship Between Gender and Disability in the Elderly People in Tehran Municipality Pension Organization
}

Helen Noei ${ }^{1}$, Robab Sahaf ${ }^{2 *}$, Ahmad Ali Akbari Kamrani ${ }^{2}$, Yadollah Abolfathi Momtaz ${ }^{2}$, Samaneh Pourhadi ${ }^{3}$, Mohsen Shati ${ }^{2}$

1. Department of Ageing, University of Social Welfare and Rehabilitation Sciences, Tehran, Iran.

2. Department of Ageing, Research Center on Ageing, University of Social Welfare and Rehabilitation Sciences, Tehran, Iran

3. Department of Community Medicine, School of Medicine, Babol University of Medical Sciences, Babol, Iran.

Received: 02 Nov. 2016

Keywords:

Disability, Elderly, Tehran City council, Sex
Accepted: 27 Jan. 2017
Cltation: Noei H, Sahaf R, Akbari Kamrani AA, Abolfathi Momtaz Y, Pourhadi S, Shati M. [The Relationship Between Gender and Disability in the Elderly People in Tehran Municipality Pension Organization (Persian)]. Iranian Journal of Ageing. 2017; 12(1): 6-17. http://dx.doi.org/10.21859/sija-12016

http://dx.doi.org/10.21859/sija-12016

\section{A B S T R A C T}

Objectives Because of increasing geriatric population in Iran, the present study aims to plan, prevent, and mitigate the complications among the retired elderly of the Tehran Municipality Pension Organization and then investigate its relation to gender.

Methods \& Materials The descriptive and analytic approach was adopted on 300 elderly subjects who were 60 years and older (Mean [SD]: 68.14[7.11] years). The subjects were selected using random sampling method. All the elderly subjects were retired members of the Tehran Municipality Administration. The data were collected by direct reference to the subjects' home and conducting interviews. The data collection tool included questionnaires with two sections. The first section consisted of the demographic, socioeconomic, and health profiles, and the second section consisted of WHO Disability Assessment Schedule 2.0 questionnaire. The obtained data were analyzed by SPSS 18.

Results Among the elderly subjects in this study, 175 (54\%) had no disability, 125 (46\%) were disabled, and $19.13 \%$ had mild disability. The average disability rate was $5.6 \%$ in females and $4.38 \%$ in males; these rates were high compared to the present rate in the society. However, the lowest rate of disability $(0.72 \%$ in males and $1.07 \%$ in females) was related to self-caring and personal health.

Conclusion In conclusion, the retired elderly of Tehran Municipality Administration showed a lower level of disability compared to those of other developed countries. However, there is an increasing need for setting up geriatric cares units as the population pattern of elderly people is rising in Iran. In addition, the rate of disability was found to be higher in females $(58.14 \%)$ compared to males $(53.31 \%)$, which shows a significant relation of disability status with gender and age $(P<0.001)$

\section{Extended Abstract}

\section{Objectives}

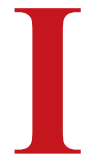

$\mathrm{n}$ recent years, there has been a sharp increase in the elderly population in Iran [1]. The aging population has been associated with the increase in the rate of disability among these elderly. Studies have shown that the extent of disability in activities and chronic diseases is inversely related to cognitive performance and quality of life [2]. Disability is a good indicator of health risk assessment in the elderly population that includes the limitation of the ability to perform social roles and activities related to the job or continuing independent life. The incidence of disability is related to several

* Corresponding Author:

Robab Sahaf, PhD

Address: Department of Ageing, Research Center on Ageing, University of Social Welfare and Rehabilitation Sciences, Tehran, Iran.

Tel: +98 (21) 2417815

E-mail: robabsahaf@gmail com 
factors. No study has been carried out in this aspect in the Tehran Municipality Pension Organization. Therefore, the present study aimed at determining the extent of disability in the elderly people and its relationship with gender.

\section{Methods \& Materials}

The Tehran Municipality Pension Organization has covered over 7400 retired employees. This study is a correlational and descriptive-analytical research carried out on 300 people aged 60 years and older (Mean [SD]: 68.14[7.11] years), who were covered by Tehran Municipality Pension Organization. These participants were selected using random sampling method and the study data were collected by referring to their homes and interviewing them. The data collection tool was a questionnaire consisted of two parts. The first part was about the demographic, economic, social, and medical information, and the second part consisted of the WHO Disability Assessment Schedule 2.0 Questionnaire (with a reliability of 0.86 and internal classification of 0.98) as follows [3]. The first part of the questionnaire included personal sociodemographic information such as age, gender, marital status, education, economic status, home ownership status, employment status, health insurance type, and chronic diseases.

WHO Disability Assessment Schedule 2.0 Questionnaire had 36 questions and measured disability in six areas. The answer to each question was given a score on a 5-point Likert-type scale. The questionnaires were completed by face-to-face interviews with the subjects. However, if the elderly were not able to respond to all questions due to their deteriorated health conditions, some questions were asked from the subject's relatives or they were interviewed over the phone. According to the World Health Organization algorithm, the raw scores were first converted to 1-100. The subjects were classified in the following way in terms of disability: subjects with a score of 1 to 4 as without disabilities; subjects with a score of 5 to 25 as having low disability; subjects with a score of 26 to 50 as having moderate disability; subjects with a score of 51 of 75 as having severe disability; and subjects with a score of 76 to 100 as having very severe disability. Of 7400 retired employees of municipality, 4300 were old people. Based on the following formula and calculation of the error percentage as well as possible rate of disability, the sample size was determined to be 280 people. However, 300 people were considered for greater certainty. The data were analyzed by SPSS (version 18). Descriptive statistics (including mean, standard deviation, and percentage) and analytical statistics (including $\mathrm{t}$ test and ANOVA) were also used. The present study was approved by the Ethics Committee of the University of Social Welfare and Rehabilitation Sciences, Iran.

\section{Results}

Among the studied elderly, 175(46\%) were without disabilities and $125(19.13 \%)$ had mild disability. The highest level of disability in the field of social presence was observed in $5.6 \%$ of women and $4.38 \%$ of men. The minimum average disability related to selfcare and personal hygiene was $0.72 \%$ in men and $1.07 \%$ in women. The present study showed that the mean disability in the age group 70-79 years was two times greater than that of the age group 60-69 years (Reference group). In the present study, there was a significant relationship between the disability level of the elderly people and chronic diseases $(\mathrm{P}=0.005)$. The disability in the age group 80 years and older was three times greater than the reference group. Also, for each year of increase in age, the disability of the elderly people has been increasing by approximately 1.1 fold.

In this study, there was a significant relationship between the level of elderly's disability and gender, and women were observed to have a higher rate of disability than men, which was consistent with other studies. 25 women $(58.14 \%)$ out of 43 were found to be disabled. Of 257 elderly men in the study, 137(53.31\%) had some degree of disability. In the present study, it was found that gender and disability had a significant relationship in the elderly. According to the study, the disability percentage in women $(58.14 \%)$ was more than the men

Table 1. The relationship between disability level and gender in the retired elderly of Tehran Municipality-2015

\begin{tabular}{cccccccccc}
\hline \multicolumn{2}{c}{ Variable } & Normal & Disability1 & Disability2 & Disability3 & Disability4 & Total & $\mathbf{X}^{2}$ & $\mathbf{P}$ \\
\hline \multirow{2}{*}{ Gender } & Female & $18(42.9)$ & $20(46.5)$ & $3(6)$ & $2(4.7)$ & $0(0)$ & $43(100)$ & \\
& Male & $120(46.7)$ & $114(44.4)$ & $16(6.2)$ & $2(0.8)$ & $5(1.9)$ & $257(100)$ & & \\
\hline
\end{tabular}


(53.31\%), and thus, disability had a significant relationship with age $(\mathrm{P}<0.001)$.

The results also showed that the severity of disability increased with older age, lower education levels, living alone and single, and lack of self-employment after retirement. Many old people suffered from at least one or more chronic and debilitating diseases.

Treatment and prevention of chronic diseases, which is the leading cause of disability in the elderly, has a significant role in the prevention of disability in the elderly. In the present study, the disability in the age group 80 years and older was greater than the other age groups, and hence, they were more dependent on others. In Table 1 the extent of mild, moderate, severe, and very severe disability (1-4) had a significant relationship with gender.

\section{Conclusion}

The elderly people in this study had lower disability rate compared with that of other developing countries. Factors such as older age, lower education, being single, chronic illnesses, and lack of self-employment after retirement have increased the severity of disability. If the municipality authorities take appropriate steps and ensure their implementation along with follow-up, chronic diseases can be controlled and elderly care home can be tracked as these factors play an instrumental role in effectively reducing disability in both genders.

In this study, the comparative analysis of disability in both genders showed that the average disability score of men was higher in the area of understanding and communicating and interacting with people. The average disability score of women was higher in the areas of moving and going around, life activities, and social presence. Possible reasons for the higher disability in women rather than men may be related to the duties of women during their life, including pregnancy, lactation, parenting, menopause, arthritis, and osteoporosis as well as the lack of relative autonomy and probably excessive responsibilities at home and work.

\section{Acknowledgments}

This research was extracted from the Gerontology MPA thesis of first author in the Department of Ageing, University of Social Welfare and Rehabilitation Sciences, Tehran, Iran.

\section{Conflict of Interest}

The authors declared no conflicts of interest. 


\title{
بررسى مقايسهاى سطح ناتوانى در زنان و مردان سالمند تحت يوشش سازمان بازنشستكَى

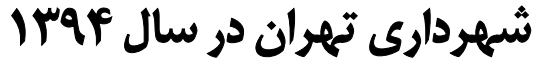

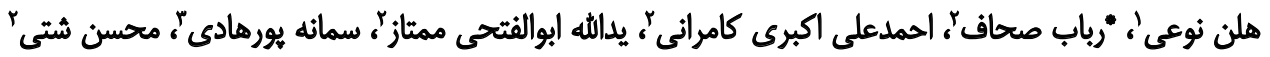 \\ 1- كروه سالمندى، دانشعاه علوم بهزيستى و توانبخشى، تهران، ايران.

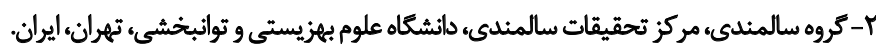

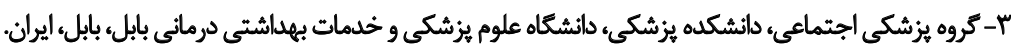

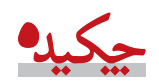

الهداف با توجه به افزايش جمعيت سالمندان در كشور، اين مطالعه با هدف تعيين ميزان ناتوانى سالمندان صورت كرفت و ارتباط آن با جنسيث انجام شد. توجنا

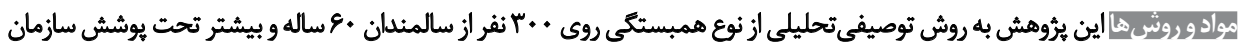

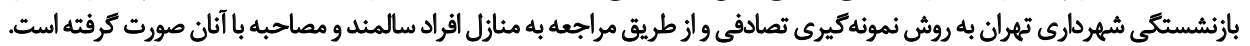

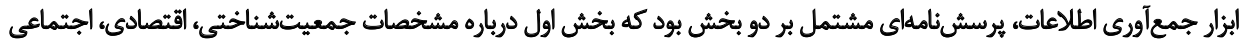

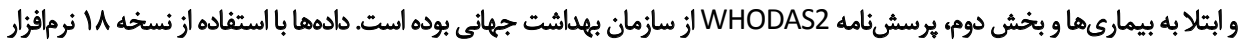
SPSS

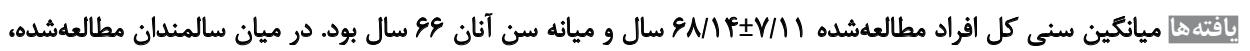

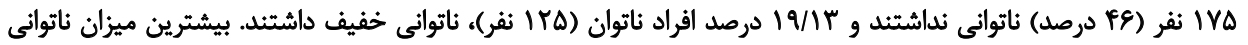

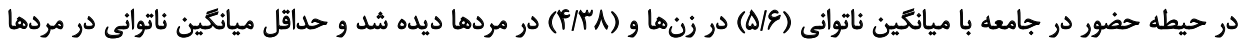

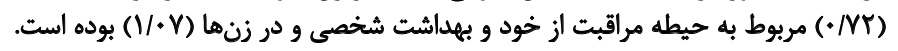

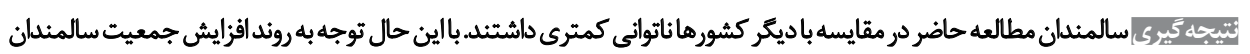

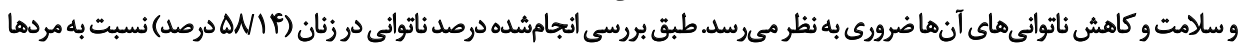

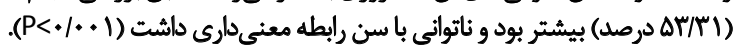

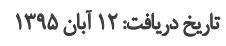

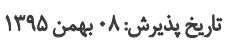

:

ثاتواني، سالمندان، شهردارى تثهران، جنسيث

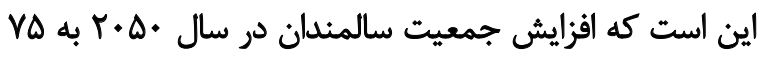

doles

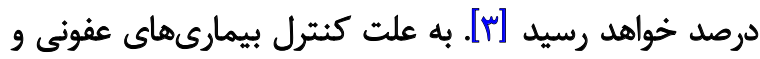

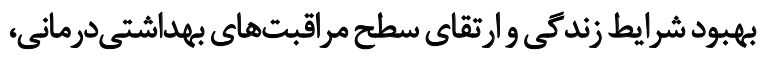

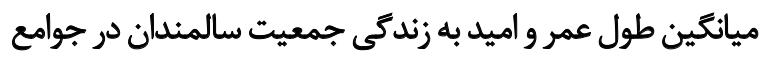

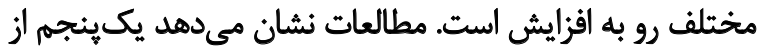

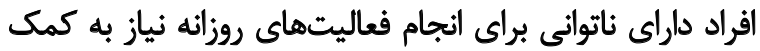

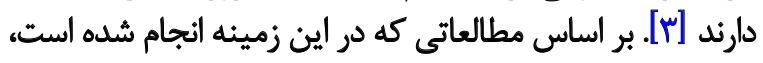

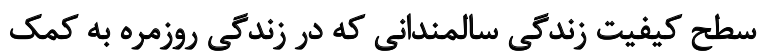

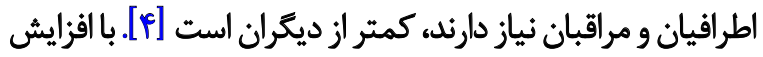

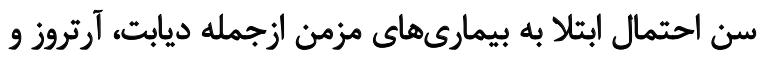

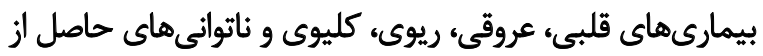

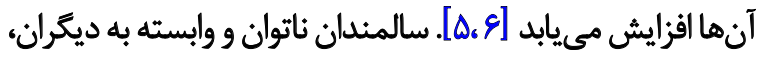
بار مالى زيادى را به خانواده و كشور وارد مي كنيند.

با توجه به افزايش سالمندان و نيازهاى خاص دوران سالمندى،

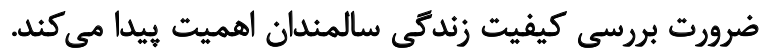

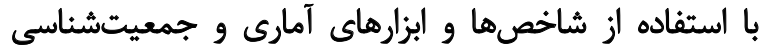

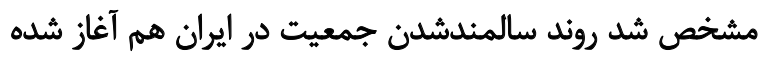

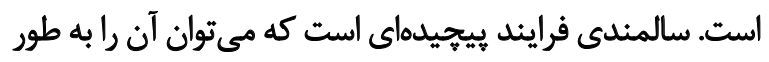

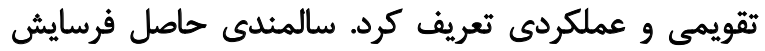

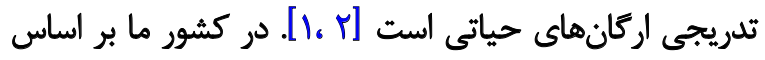

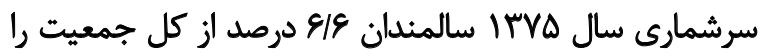

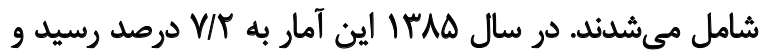

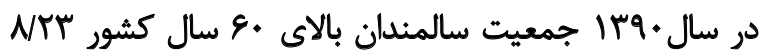

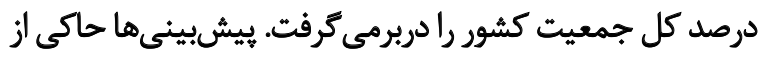

: نويسنده مسئول:

دكتر رياب صحاف نوان

نشانى: تهران، دانشعَاه علوم بهزيستى و توانبخشى، مركز تحقيقات سالمندى، كروه سالمندى.

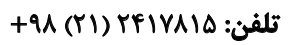
بست الكترونيكى: robabsahaf@gmail com 
سالمندى مرتبط در كشور به نتايج اين يُروهش بسيار نياز است.

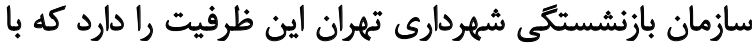

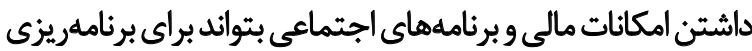

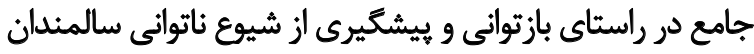

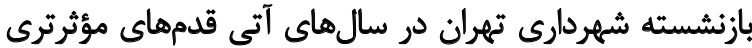

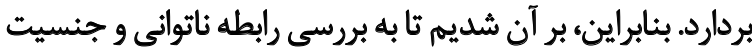

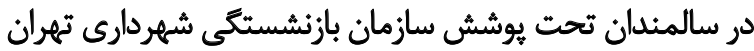

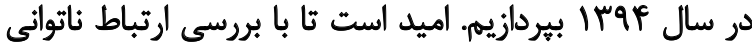

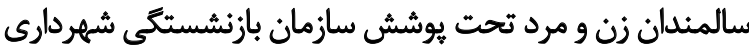

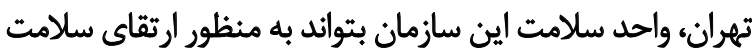

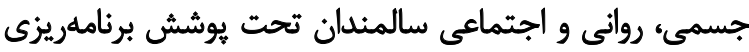

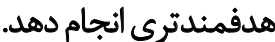
روش مطالعه

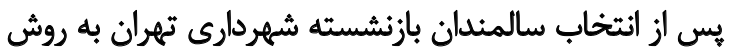

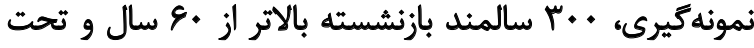

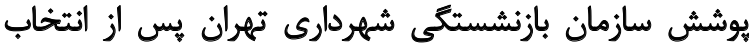

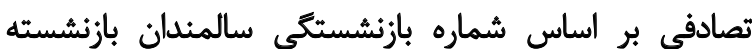

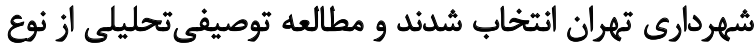

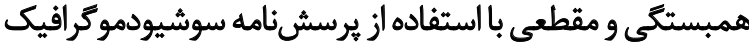

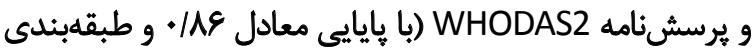

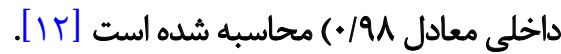

ا. يرسشنامه اول دربركيرنده اطلاعات سوشيودموكرافيك

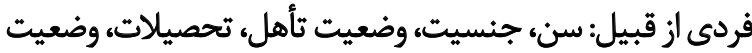

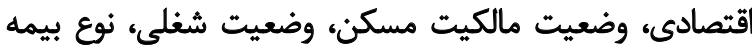

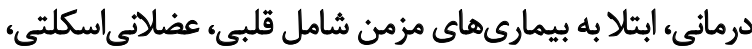

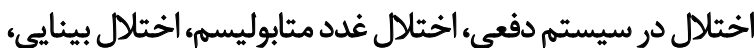
اختلال شنوايى و بيمارى عفونى بود.

ك. در بررسى شيوع ناتوانى، از توان فردى سالمند براى انجام

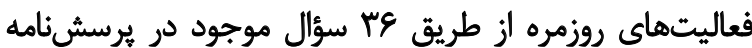

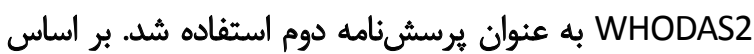

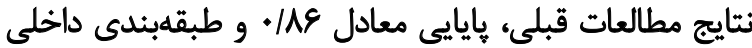

معادل 194/ محاسبه شده است [1 I I]

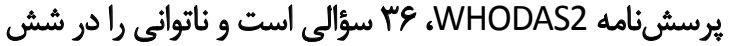

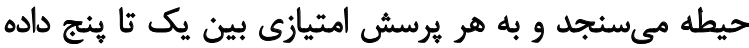

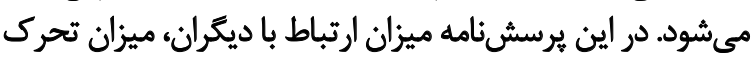

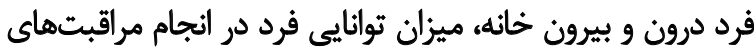

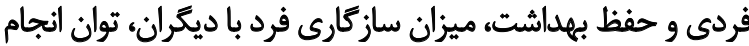

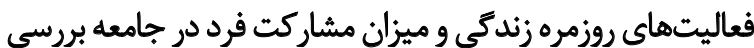

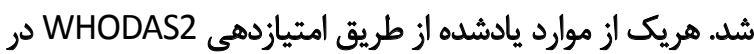

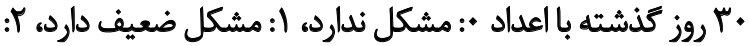

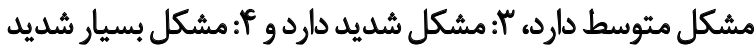

به طور كلى، ناتوانى شاخص خوبى براي سنجش ريسك سلامت

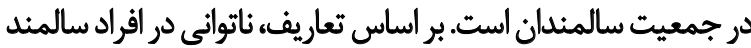

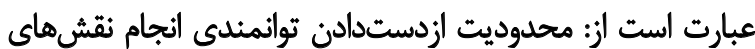

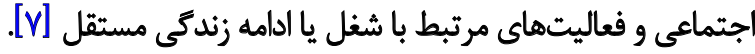

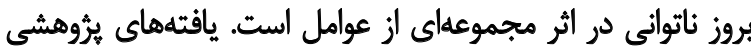

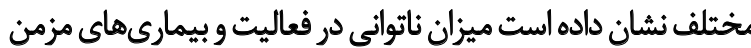

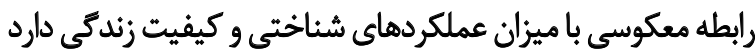

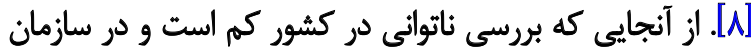

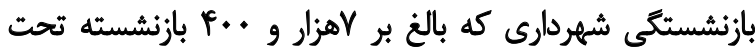

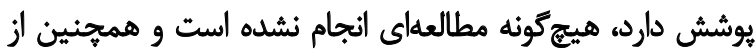

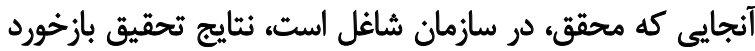

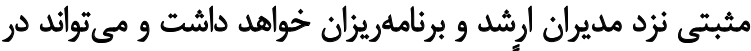
بهبود ناتواني خصوصاً در جنس شاخص مؤثر باشدي

در بررسى حاضر، بين سطح ناتوانى سالمندان تحت يوشي

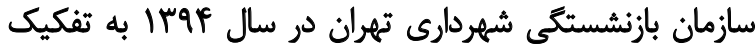

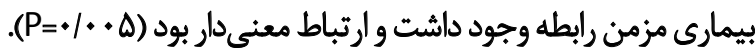

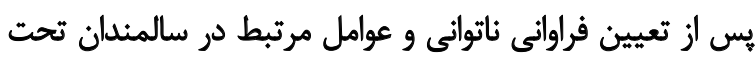

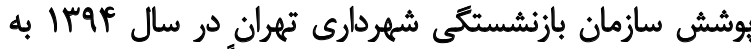

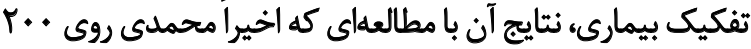

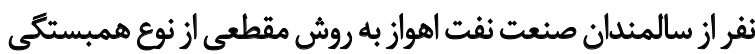

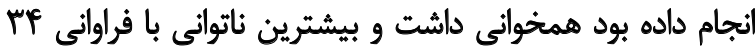

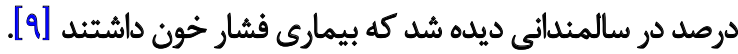

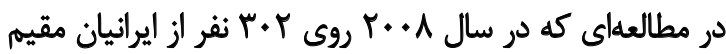

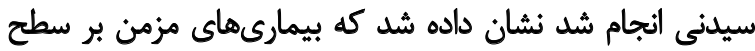

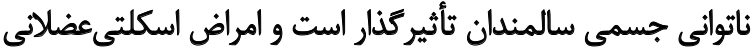

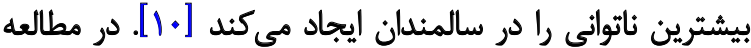

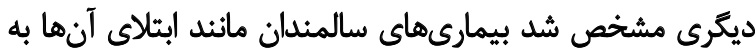

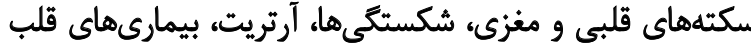

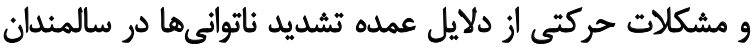

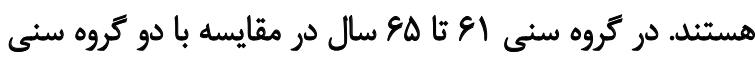

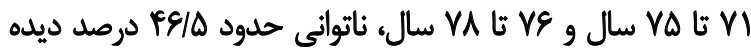

شد [11]

در سالمندان تحت يوشش شهردارى تهران افراد مبتلا به

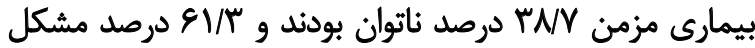

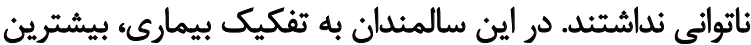

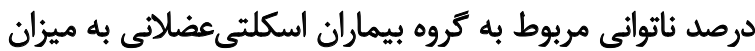

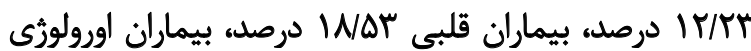

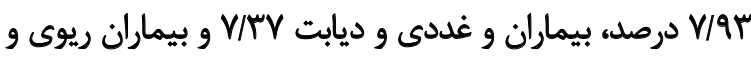

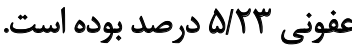

تاكنون در سازمان بازنشستكى شهردارى تهران هيج يثروهشى دئي

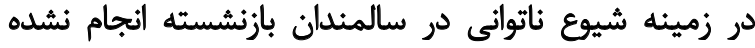
است. براى برنامهريزىهاى آينده و ارائه خدمات و مرانينه 
تحليلى تى تست و آنوا' نيز استفاده شد.

يافتهها

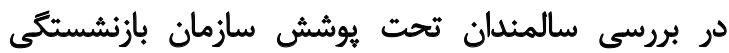

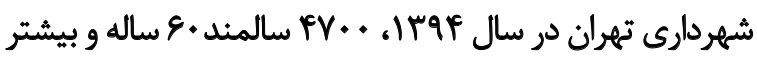

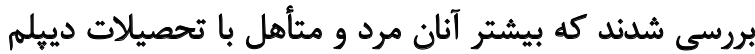

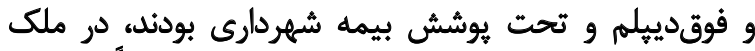

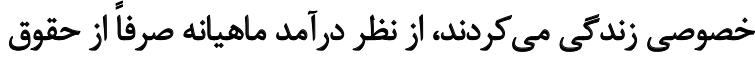

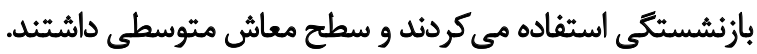

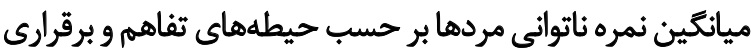

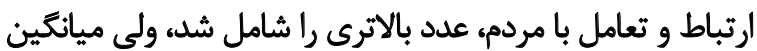

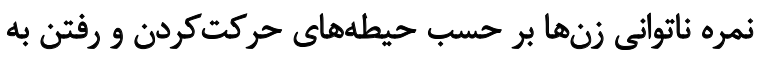

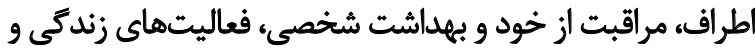

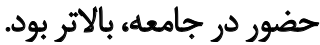

ميانكين سنى سالمندان تحت يوشش سازمان بران بازنشستى برائى شهردارى تهران در سال

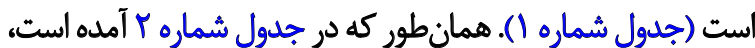

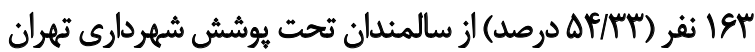

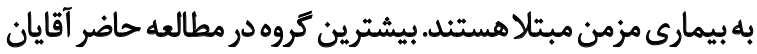

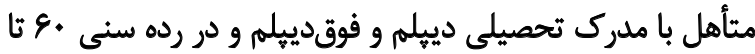

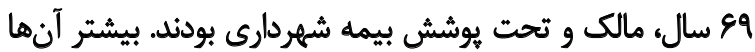

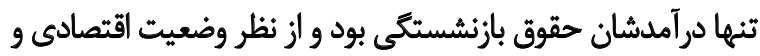

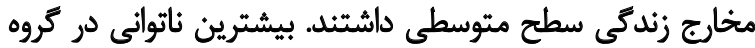

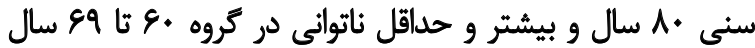

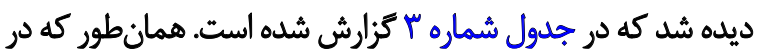

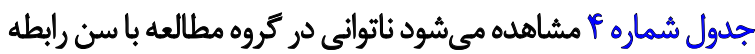
معنى دارى داشت و با افزايش سن بر ميزان ناتوانى افزوده مي شيد . $(\mathrm{P}<\cdot 1 \cdot \bullet 1)$

بر اساس نتايج ذكرشده در جدول شماره ه ناتوانى در زنها
دارد، امتيازبندى و جمع و تحليل شد. امتياز بالانمايانكر نياز بيشتر

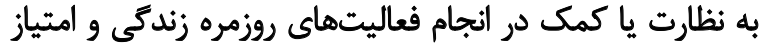

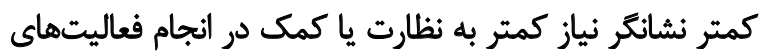

$$
\text { روزمره زندگى بود. }
$$

Pواتحت سالمند بازنشسته •\& ساله و بالاتر كه در سال

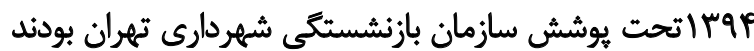

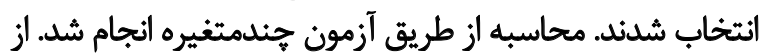

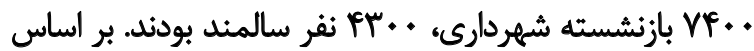

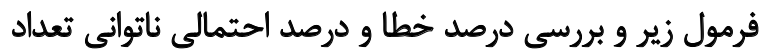

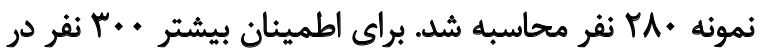
نمونهكيرى دخالت داده شدند.

$$
n=\frac{z^{2}(1-\alpha / 2) \times P \alpha(1-P)}{d^{2}}
$$

يس از تكميل، يرسشنامه

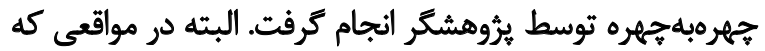

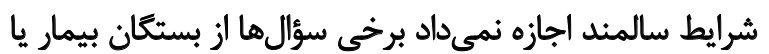
تلفنى يرسيده شد.

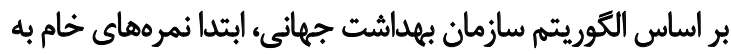

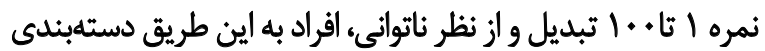

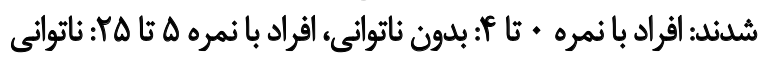

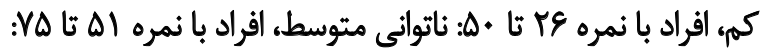

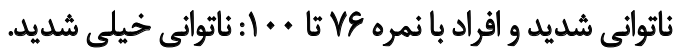

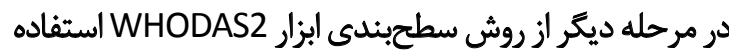

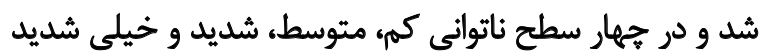

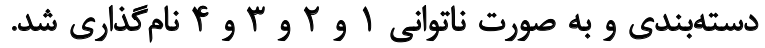

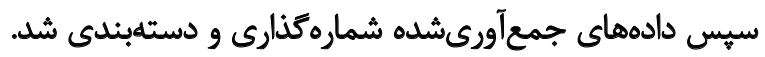

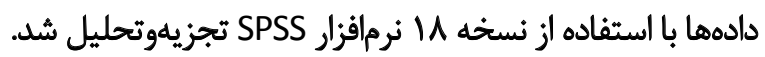

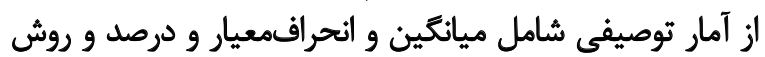

\begin{tabular}{|c|c|c|}
\hline انحر|فمعيار & ميائكين & مثغير \\
\hline$V / 11$ & ENIF & سن \\
\hline
\end{tabular}

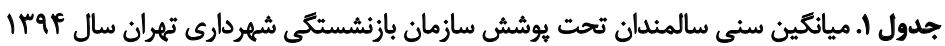

$\stackrel{1}{2}$

\begin{tabular}{|c|c|c|c|c|}
\hline مجموع & درصد & تعداد & \multicolumn{2}{|c|}{ متغير } \\
\hline \multirow{2}{*}{$r+\cdots(1 \cdots)$} & $\Delta F / M r$ & Tr & دارد & \multirow{2}{*}{ بيمارى مزمن } \\
\hline & $P \Delta / \& V$ & Irv & نلارد & \\
\hline
\end{tabular}

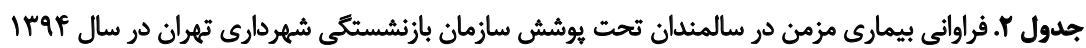

كالم 
جدول ז. ارتباط ناتوانى با سن در سالمندان بازنشسته شهردارى تهران -سال Irqf

\begin{tabular}{|c|c|c|c|c|c|c|}
\hline$P$ & $\mathbf{X}^{2}$ & مجموع & ناتواتى ندارد & ناتوانى دارد & \multicolumn{2}{|c|}{ نام متغير } \\
\hline \multirow{3}{*}{$\cdot|+\cdot 1\rangle$} & \multirow{3}{*}{$19 / \pi T$} & $(1.0) 198$ & $(\Delta \Delta / 1) 1 \cdot \Lambda$ & (fie/a)M & $8 \cdot-89$ & \multirow{3}{*}{ سن } \\
\hline & & $(1.0) \mathrm{ra}$ & $(r+/ A) r r$ & $(E q / T) \Delta F$ & $r=-v q$ & \\
\hline & & (1+. & $(M / N) E$ & $(N \& / q) r$. & •1 و بالاتر & \\
\hline
\end{tabular}

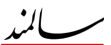

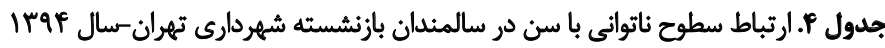

\begin{tabular}{|c|c|c|c|c|c|c|c|c|c|}
\hline $\mathbf{P}$ & $x^{2}$ & هجموع & ناتوانى F & ناتوانى r & ناتوانى Y & ناتوانى & ترمال & نام هتغير & \\
\hline \multirow{3}{*}{$\cdot 1 \cdot+1>$} & \multirow{3}{*}{$\triangle N M F$} & $(1.0) 118$ & $(\cdot) \cdot$ & $(\cdot) \cdot$ & $(T / \varepsilon) V$ & $(r / F)$ (N) & $(\Delta \omega / 1)) \cdot A$ & $q \cdot-8 q$ & \multirow{3}{*}{ سن } \\
\hline & & $(1 \cdot \cdot) \mathrm{rs}$ & $(r / \varepsilon) r$ & $(T / g) r$ & $(N / V)$ & $(\Delta F / f)+\varepsilon$ & $(Y \cdot / \Lambda) M r$ & $r=-r q$ & \\
\hline & & $(1+0) \times 8$ & $\left(r^{\mu}\right) 11 / \Delta$ & $(V / V)$ & $(K M / N) E$ & $(m e / 8)^{q}$ & $(\Pi M / N)$ & •1 و بالاتر & \\
\hline
\end{tabular}

il

خود اختصاص داده است. البته به علت وظيفه زنان در امور منزل و ونان

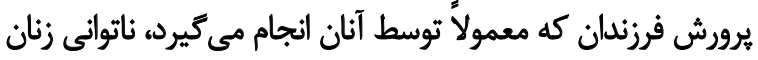

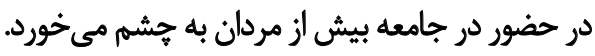

$\stackrel{\leftrightarrow}{*}$

در بررسى حاضر با هدف بررسى وضعيت موجود سالمندان

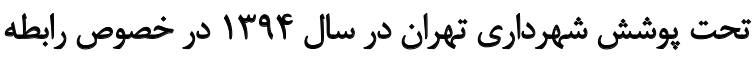

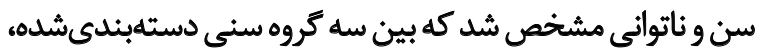

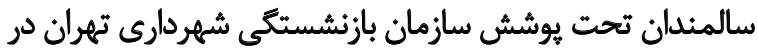

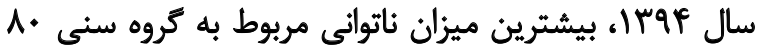

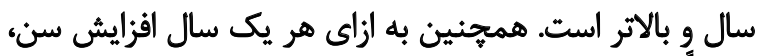

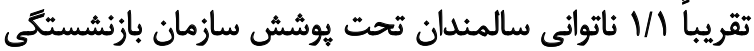

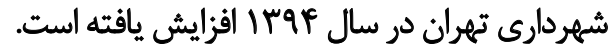

بيشتر از مردها ديده شد و اين موضوع مي تواند به علت زايمان،

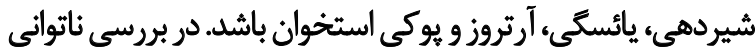

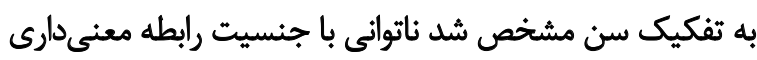

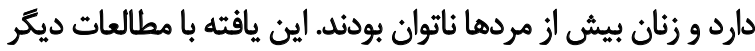

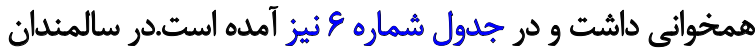

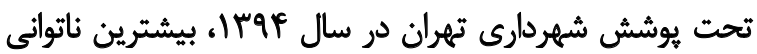

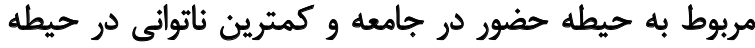
مراقبت از خود و بهداشت شخصى بود (جدول شماره V).

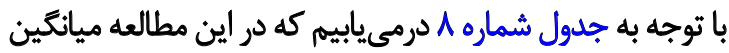

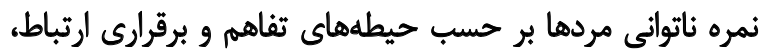

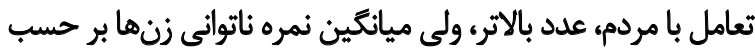

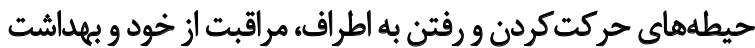

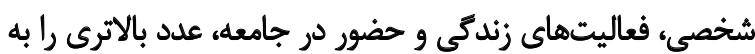

جدول هـ ارتباط ناتوانى با جنسيت در سالمندان بازنشسته شهردارى تهران-سال IF

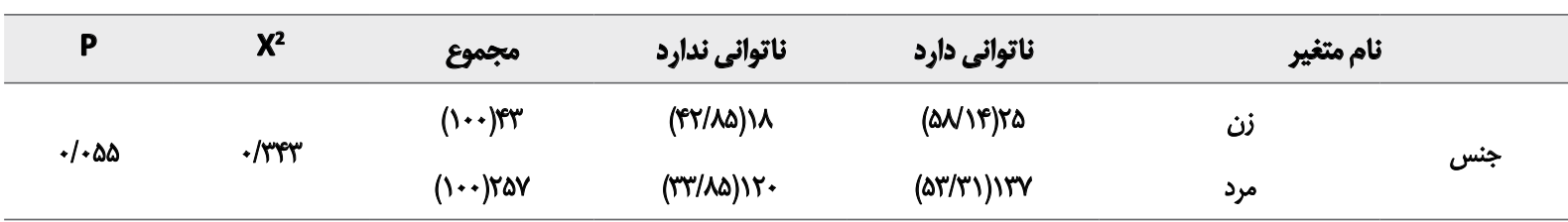

جدول \&. ارتباط سطوح ناتواني با جنسيت در سالمندان بازنشسته شهردارى تهران-سال ITFF

\begin{tabular}{|c|c|c|c|c|c|c|c|c|}
\hline $\mathbf{P}$ & $X^{2}$ & هجموع & ناتواني f & ناتواتيى & ثاتوائى T & ناتواتى ا & نرمال & نام متغير \\
\hline \multirow{2}{*}{1.50} & \multirow{2}{*}{$\Delta / \pi m$} & & & $(r / M) r$ & $(8)^{r}$ & & & \multirow{2}{*}{ جنسيت } \\
\hline & & $(1+$.$) rar$ & $(1 / 9) \Delta$ & $(\cdot / A) r$ & $(\varepsilon / T) \backslash \varepsilon$ & $(f f / f) I I f$ & $(f \in / V)$ Ir. & \\
\hline
\end{tabular}

旔 
جدول V. ميانكين ناتواني درحيطهاي تفاهم و برقرارى ارتباط، حركت، مراقبت از خود، تعامل با مردم، فعاليتهاى زندكى، حضور در جامعه در سالمندان تحت

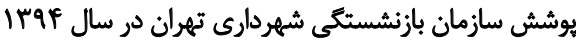

\begin{tabular}{|c|c|c|c|c|c|c|c|c|c|}
\hline تعداد كل & نرمد از كل & $\mathbf{P}$ & $X+S D$ & ميانكين درصد & انحرافمعيار & مياتكين & داهنه & دامنه مرجع & متغير مورد سنجش \\
\hline r.. & $f$ &.$/ 4 r$ & $\mid / 1 \pm r / q 8$ & W/TF & $|r / T|$ & $r / N$ & $\cdot-r \cdot$ & $\cdot-r \cdot$ & برقرارى ارتباط و تثفاهم \\
\hline r.. & r &.$/ N r$ & $r / M I t / T r$ & $r(A)$ & $|E / \Delta|$ & NFF & - tra & - ra & حركت كردن و رفتن به \\
\hline$r+$. & $9 / \mu$ & עr/. & $\cdot M \pm T / V$ & I/T & $\mid r / F \Delta$ & $r / A \mu$ & +-18 & $\cdot-r \cdot$ & مراقبت ازخصود (بهداشت \\
\hline$r$ & $1+/ r$ & .119 & $V / \wedge q \pm r / \cdot V$ & $Y(\Delta)$ & $1 r / 4 q$ & V/ar & . $-r$. & - TA & تعامل با مردم \\
\hline$r+$. & $\Lambda$ & $\cdot / r A$ & $p / p q \pm F / \cdot A$ & $r / r^{\mu}$ & IV/Fe & $11 / r$ & $4-M$ & $-t+$ & فعاليتهاى زندكى \\
\hline r.. & 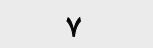 & .119 & $\Delta / e_{ \pm}+V / N$ & $F / \Delta F$ & $19 / 90$ & $|r / r|$ & - $-M T$ & $-t r$. & حضور در جامعه \\
\hline
\end{tabular}

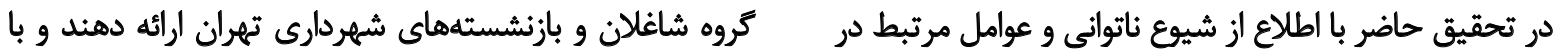

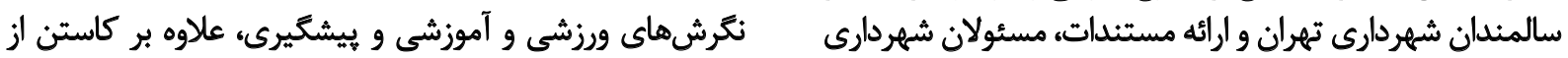

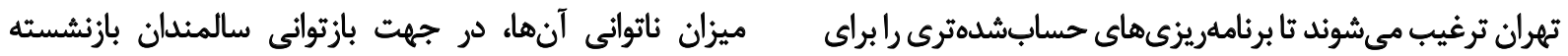
جدول A. مقايسه ميانكين و انحرافمعيار نمرات ناتوانى در سالمندان بازنشسته شهردارى تهران به تفكيك حيطهاى تفاهم و برقرارى ارتباط، حركت، مراقبت از

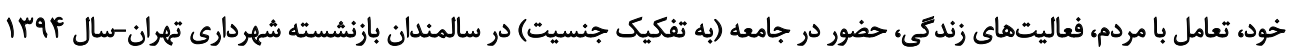

\begin{tabular}{|c|c|c|c|c|c|c|c|}
\hline تعداد & $X \pm S D$ & $\mathbf{P}$ & انحرافمعيار & مياتكين & جنس & دامثه & متغير مورد سنجش \\
\hline $\begin{array}{l}\text { TAV } \\
\text { rT }\end{array}$ & 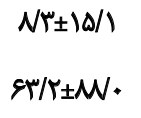 & $\mathrm{PH} /$. & $\begin{array}{l}\text { A)/r } \\
8 \pi / r\end{array}$ & $\begin{array}{l}1090 / 1 \\
M .\end{array}$ & زمد & $r+-$ & برقرارى ارتباط و تثاهم \\
\hline $\begin{array}{l}\text { rov } \\
\text { re }\end{array}$ & $\begin{array}{c}Q / r \pm \cdot r / r \\
\Delta \pm \Delta V / r\end{array}$ & $\mid r y$. & $\begin{array}{l}q / \mu \\
0\end{array}$ & $\begin{array}{l}\cdot F / T \\
\Delta V / T\end{array}$ & زرد & $r \Delta=$. & حركت كردن و رفتن \\
\hline $\begin{array}{l}\text { ror } \\
\text { it }\end{array}$ & $\begin{array}{l}V / T \pm V /+ \\
\Delta \Delta / T_{ \pm} \cdot V /\end{array}$ & $\mathrm{mTT} /$ & $\begin{array}{l}V / r \\
\Delta \Delta / r\end{array}$ & $\begin{array}{l}n / . \\
+V / 1\end{array}$ & نرد & $r+-\cdot$ & 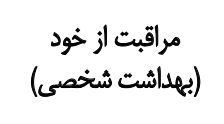 \\
\hline $\begin{array}{l}\text { TAY } \\
\text { Pr }\end{array}$ & $\begin{array}{l}|W / W \pm Q| \\
E N T \pm N \mid\end{array}$ & 1QW. & $\begin{array}{l}\text { ITQ/Y } \\
\text { \&NY }\end{array}$ & $\begin{array}{l}9.11 \\
1.11\end{array}$ & زرد & TA-. & تعامل با هردم \\
\hline $\begin{array}{l}\text { rar } \\
\mathrm{rT}\end{array}$ & $\begin{array}{l}N \varepsilon_{ \pm}+N F \\
V q / V \pm 1 \& / \Delta\end{array}$ & $\mathrm{rAr} /$ & $\begin{array}{l}\text { Ne/s } \\
\mathrm{ra/Y}\end{array}$ & $\begin{array}{l}\text { WNF } \\
1 \% / 0\end{array}$ & زرد & $f+-\cdot$ & فعاليتهاى زندكى \\
\hline $\begin{array}{l}\text { TAY } \\
\text { RT }\end{array}$ & $\begin{array}{l}W / V_{ \pm} r / \mathrm{s} \\
\varphi q / \mathrm{A}+\varphi / \mathrm{\varphi}\end{array}$ & $19 \% /$. & $\begin{array}{l}W / V \\
P q / A\end{array}$ & $\begin{array}{l}\text { relo } \\
\text { p/s. }\end{array}$ & زهرد & p.-. & حضور در جامعه \\
\hline
\end{tabular}




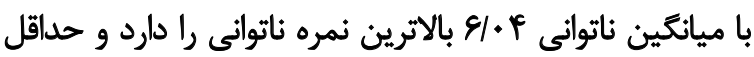

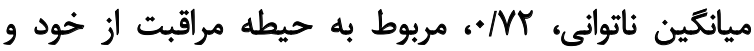
بهداشت شخصى است.

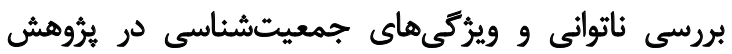

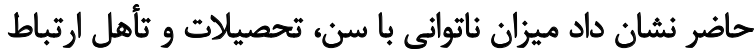

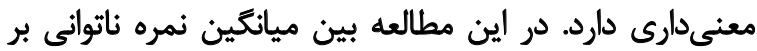

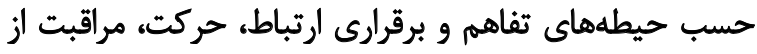

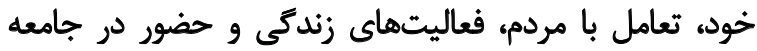

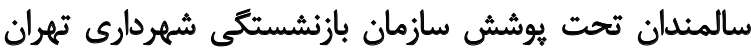

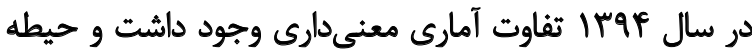

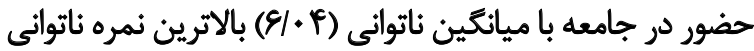

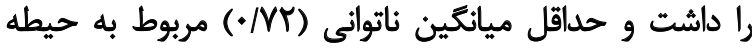

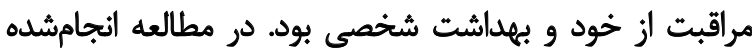

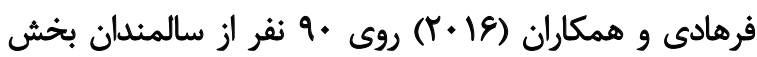

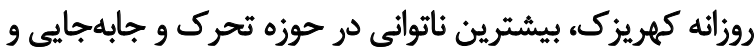

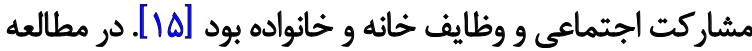

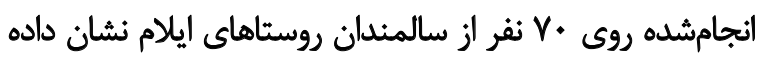

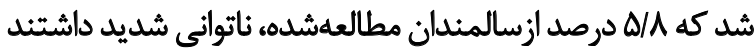

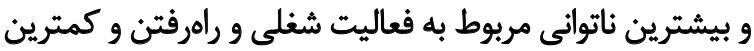

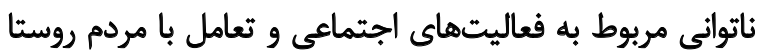

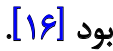

\section{نتيجلَيرى نمهايى}

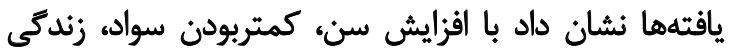

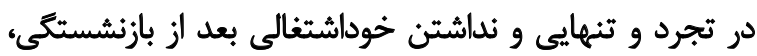

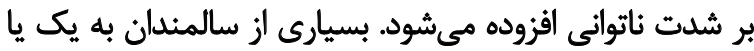

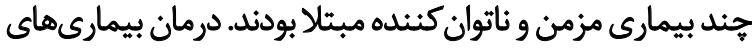

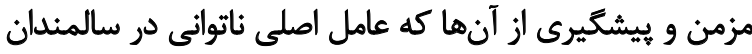

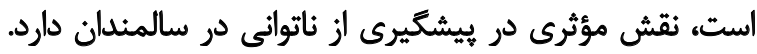

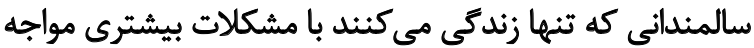

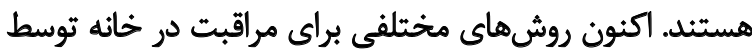

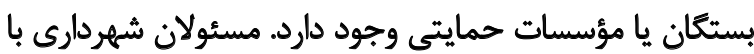
انتخاب روشهاى مناسب، سياستهاى ابداعى رادي رادر اين راستا به كار بستند. - كاب روش

در اين مطالعه ميانكين نمره ناتوانى مردها بر حسب حيطههاى

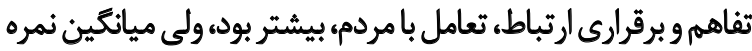

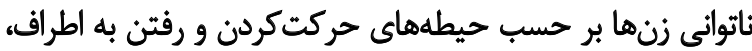

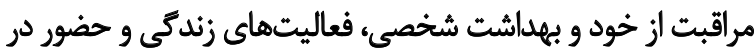

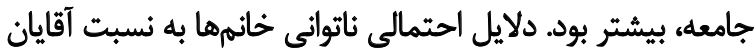

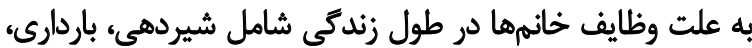
بجهذدارى، يائسكى، آرتروز، يوكى استخوان و غيره است.
كامهاى مؤثرثترى بردارند.

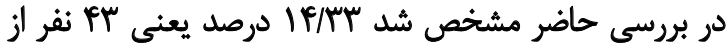

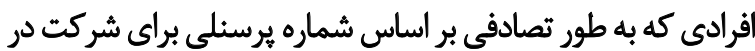

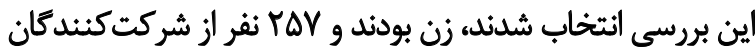

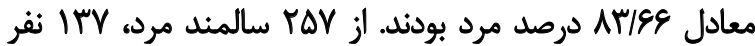

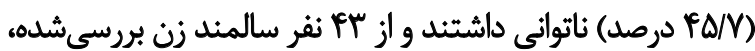

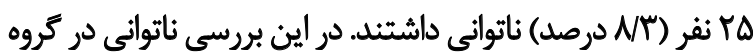

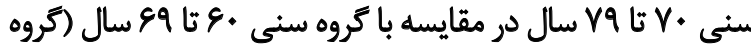
(رفرنس) بيش از دو برابر بود.

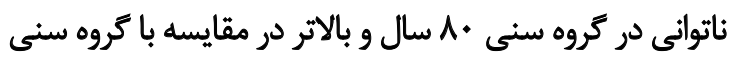

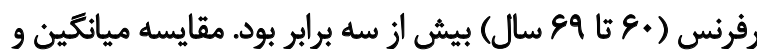

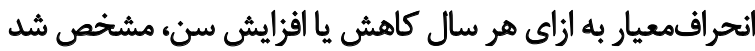

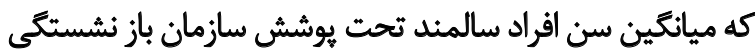

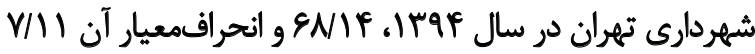

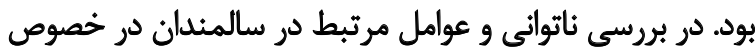

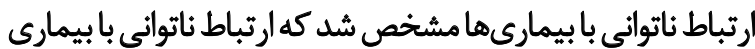

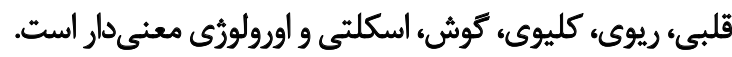

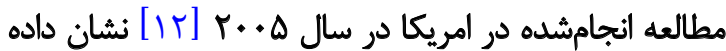

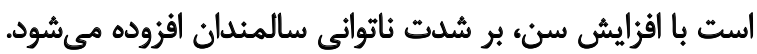

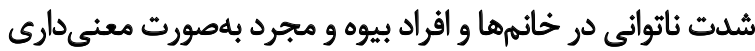

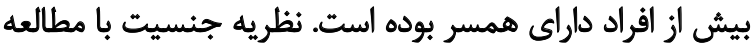
حاضر همخواني داشت.

بين سطح ناتوانى سالمندان در ئروهش حاضر، به تفكيك

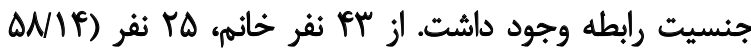

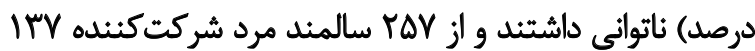

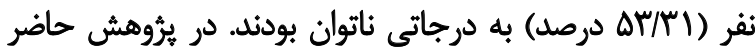

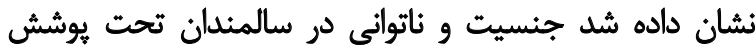

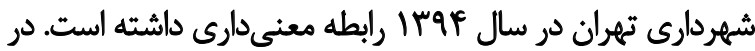

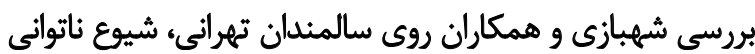

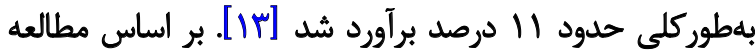

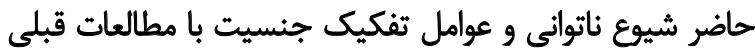

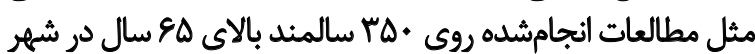

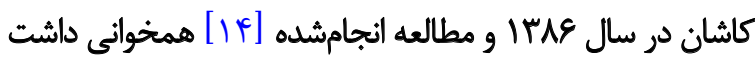

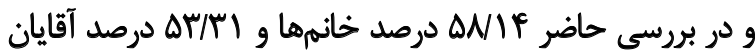
ناتوان بودند و ارتباط ناتوانى با جنسيت معنى دار بردي بودان

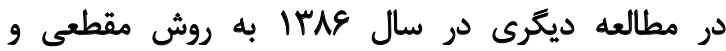

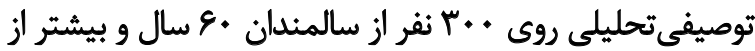

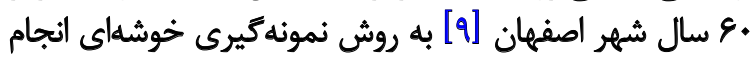

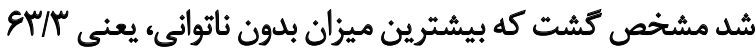

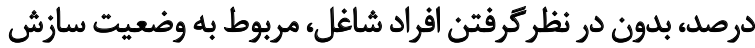

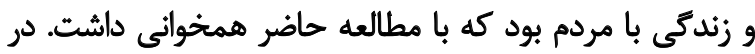

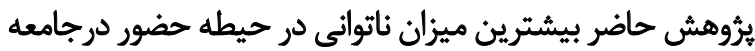




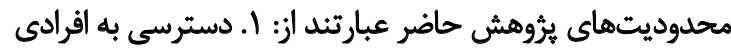

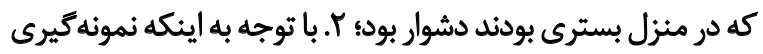

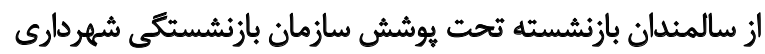

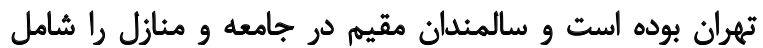

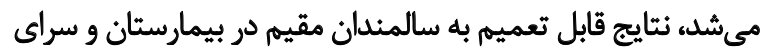

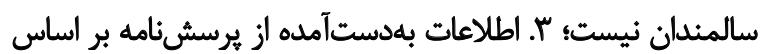

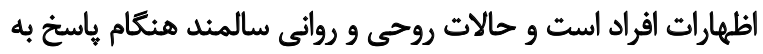

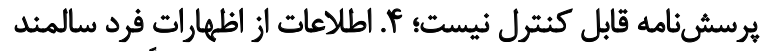

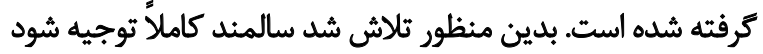

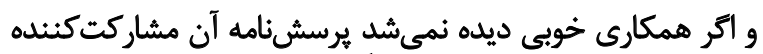

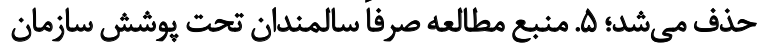

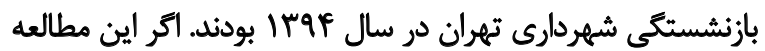

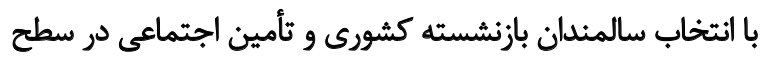
وسيعترى انجام شود، نتايج بهترى به دست خواهد آمدان

\section{بيشنهادها}

بيشنهادهاى نويسندكان براى مطالعات آتى عبارتند از: ا. تحقيق

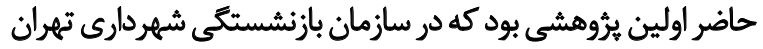

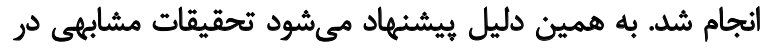

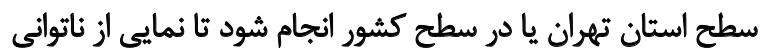

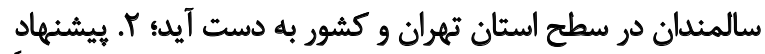

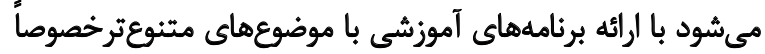

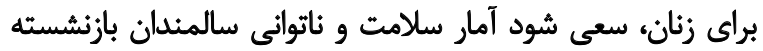

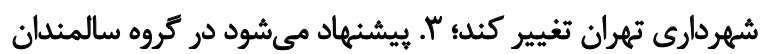

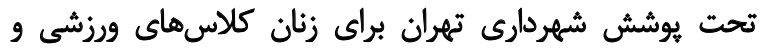

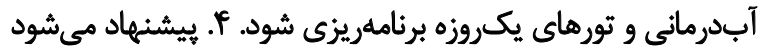

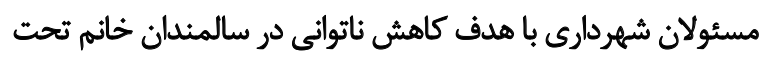

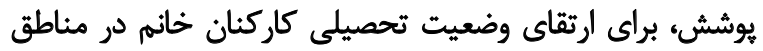
r بكانه شهردارى تهران همت كمارئد

$$
\text { تشكير وقبدرفاني }
$$

اين مقاله از باياننامه MPH سالمندى خانم هلن نوعى در دانشكاه

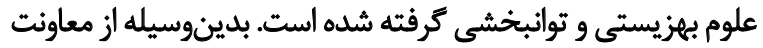

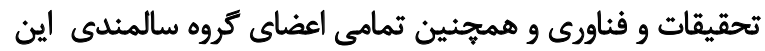

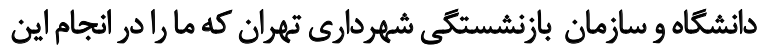

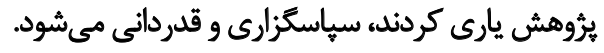




\section{References}

[1] Ministry of Health and Medical Education. [Estimating life expectancy and prediction of Iranian population (Persian)]. Tehran: Ministry of Health and Medical Education; 2006.

[2] Khoshbin S, Eshrati B, Azizabadi Farahani A. [Report of the elderly status of Iran in 2002 (Persian)]. Tehran: Ministry of Health and Medical Education; 2007.

[3] Khoshbin, Eshrati B, Azizabadi Farahani A. [Report of the elderly population of Iran in 1998 (Persian)]. Tehran: Ministry of Health and Medical Education; 1998.

[4] Morovati Sharifababd, MA, Hosseini Sharifababd, M. [Assessment of activities of daily living in elderly 65 years old in Yazd (Persian)]. Journal of Shahid Sadoughi University of Medical Sciences. 2000; 8(4):46-53.

[5] Avlund K, Due P, Holstein BE, Heikkinen RL, Berg S. Changes in social relations in old age: Are they influenced by functional ability. Aging Clinical and Experimental Research. 2002; 14(3):56-64. PMID: 12475134

[6] World Health Organization. Active aging [Ministry of Health and Medical Education Persian trans]. Tehran: Ministry of Health and Medical Education; 2003.

[7] Shirazikhah M, Mousavi M, Sahaf R, Sarmadi M. [Consequence of changes in the elderly people population: Elderly women in Iran (Persian)]. Life Science Journal. 2012; 9(4):869-77.

[8] Naghavi M. [Portrait of death in four provinces of Iran 1999 (Persian)]. Tehran: Termeh; 2000.

[9] Saeidimehr S, Geravandi S, Izadmehr A, Mohammadi MJ. [Relationship between the "Quality of Life" and symptoms of depression among older adults (Persian)]. Iranian Journal of Ageing. 2016; 11(1):90-9.

[10] Alizadeh-Khoei M. Assessing factors in utilisation of health services and community aged care services by the Iranian elderly living in the Sydney metropolitan area: Acculturation aged care [PhD thesis]. Sydney: University of Sydney; 2008.

[11] Shahbazi MR, Mirkhani M, Hatamizadeh N, Rahgozar M. [Disability assessments in Tehranian elderly 2007 (Persian)]. Iranian Journal of Ageing. 2008; 3(3-4):84-92

[12] Adib Hajbagheri M. [Geriatic disability related factors (Persian)]. Iranian Journal of Ageing. 2008; 3(2):547-55.

[13] Shahbazi MR, Foroughan M, Salman Roghani R, Rahgozar M. [The relationship between disability and variables of depression, cognitive status, and morale among older people (Persian)]. Iranian Journal of Ageing. 2016; 11(1):132-141

[14] Salive ME, Guralnik JM. Disability outcomes of chronic disease and their implications for public health. Baltimore: Johns Hopkins University Press; 1997.

[15] Farhadi A, Foroughan M, Mohammadi F, Rassouli M, Sadegh Moghadam L, Nazari S, et al. [Caregiving appraisal in family caregivers of older adults (Persian)]. Iranian Journal of Ageing. 2016; 11(1):8-19

[16] Mozafari M, Salimi E, Bastami MR, Azami M, Borji M. [Disability status in the rural older adults in llam (Persian)]. Journal of Gerontology. 2016; 1(1):48-54. doi: 10.18869/acadpub.joge.1.1.64 
